\title{
Estrogen and progestin regulate metastasis through the PI3K/AKT pathway in human ovarian cancer
}

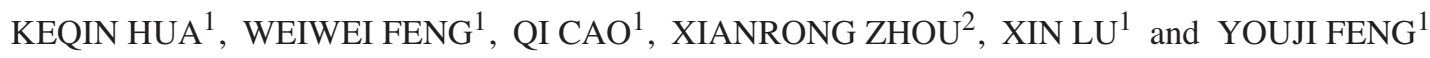 \\ Departments of ${ }^{1}$ Gynecology, ${ }^{2}$ Pathology, The Obstetrics and \\ Gynecology Hospital of Fudan University, Shanghai, P.R. China
}

Received April 14, 2008; Accepted June 12, 2008

DOI: 10.3892/ijo_00000083

\begin{abstract}
Estrogen and progestin are involved in ovarian carcinogenesis. Change in nm23-H1 expression and the PIK3/ AKT pathway are involved in carcinogenesis, development, invasion and metastasis of ovarian cancers. Therefore, it is critical to understand the signaling pathways that regulate hormone-induced cell migration and invasion in ovarian cancer. We investigated nm23-H1, AKT and pAKT expression by using immunohistochemical staining in ovarian clear cell adenocarcinoma, ovarian benign, borderline and malignant serous tumors and analyzed their relationship with prognostic factors. Using ES-2 and SKOV-3 ovarian cancer cell lines, we studied the modulation of estrogen and progestin on cell migration and invasion as well as their effect on AKT, pAKT and $\mathrm{nm} 23-\mathrm{H} 1$ expression. Furthermore, the signaling pathways were examined using pharmacological inhibitors (LY294002 and PD98059) or AKT siRNA combined with estrogen or progestin in the two cell lines. Weak nm23-H1 and high AKT and pAKT expression was observed in ovarian serous adenocarcinoma and ovarian clear cell adenocarcinoma. Our data demonstrated that the expression of nm23-H1 was negatively correlated with tumor stage and grade and lymph node metastasis, whereas the expression of AKT/pAKT was positively correlated with these clinic factors. Estrogen upregulated pAKT expression and reduced nm23-H1 expression, which ultimately resulted in increased cell migration and invasion. In contrast, progestin reduced pAKT expression and increased nm23-H1 expression, which inhibited cell migration. PIK3 kinase inhibitor LY294002 antagonized the effect of estrogen. On the other hand, it reinforced the effect of progestin. Our data suggest that AKT and pAKT are unfavorable prognostic factors for ovarian serous adenocarcinoma and clear cell carcinomas whereas nm23-H1 expression predicates favorable patient prognosis. Estrogen down-regulates nm23-
\end{abstract}

Correspondence to: Dr Keqin Hua or Dr Youji Feng, Department of Gynecology, The Obstetrics and Gynecology Hospital of Fudan University, 419 FangXie Road, Shanghai 200011, P.R. China E-mail: huakeqin@126.com; fengyj4806@sohu.com

Key words: ovarian cancer, estrogen, progestin, nm23-H1, phosphatidylinositol 3-kinase pathway
H1 expression and promotes cell migration and invasion by activating the PIK3/AKT pathway. Progestin has an opposing effect.

\section{Introduction}

Epithelial cancers are the most common ovarian malignancies. Among them, clear cell carcinoma and serous adenocarcinoma are more aggressive, tend to metastasize and are associated with poor patient prognosis $(1,2)$. Many factors facilitate the migration and invasion of tumor cells, such as tumor secretions, growth factors, the extracellular matrix and metabolites of metastatic target organs. The ovary produces sex steroids. A previous study revealed that postmenopausal estrogen replacement therapy without progestin was a risk factor of carcinogenesis of ovarian clear cell carcinoma, whereas hormone replacement therapy (HRT) with progestin did not increase the incidence of ovarian cancer (1). On the other hand, oral combination contraceptives (estrogen combined with progestin) decreased the incidence of ovarian cancer $(3,4)$. In addition, a high dose of progestin reduced the risk of ovarian cancer to a greater extent (5), suggesting the protective effect of progestin. But the correlation between sex steroids and ovarian carcinogenesis is still unknown, and the mechanism has not yet been investigated.

It is known that sex steroids are implicated in controlling migration and invasion by two pathways (transcription and non-transcription). Both estrogen receptor (ER) $\alpha$ and ERß induce gene transcription through these pathways. In ERpositive ovarian cancer cells, estrogen activates the phosphatidylinositol 3-kinase/protein-kinase B (PI3K/PKB) signaling pathway through a non-transcription pathway $(6,7)$. Previous research suggests that the PIK3 pathway is important in ovarian carcinogenesis (8). Overexpression of AKT was found in ovarian cancer, implying that it might be involved in ovarian carcinogenesis (9-11). In order to identify whether estrogen stimulates the cellular signal transduction pathway, we observed the effects of estrogen and progestin on migration and invasion in ovarian cancer cell lines ES-2 and SKOV-3, and investigated the links between sex steroids and the $\mathrm{PI} 3 \mathrm{~K} / \mathrm{AKT}$ pathway. Metastasis is a multi-step process involving host tumor interactions. Sex steroids could regulate cell migration and invasion by affecting metastasis-related genes. $\mathrm{nm} 23-\mathrm{H} 1$ is a tumor suppressor gene and is positively expressed in normal cells. Loss of $\mathrm{nm} 23-\mathrm{H} 1$ expression in 
cancers suggests that $\mathrm{nm} 23-\mathrm{H} 1$ may be involved in carcinogenesis, development, invasion and metastasis (12). Decreased nm23-H1 expression has been reported to correlate with clinical tumor stage, grade, lymph node metastasis and patient survival in ovarian serous and clear cell carcinomas (13-15). To date, the mechanism involved in the regulation of tumor metastasis-related genes by steroids via the signal transduction pathway remains unclear .

In the present study, our first aim was to characterize the expression of AKT, phosphorylated AKT (pAKT) and nm23$\mathrm{H} 1$ in benign, borderline serous adenoma, serous adenocarcinoma as well as clear cell carcinoma, in order to analyze the relationship between protein expression and prognostic factors. Secondly, using the ES-2 and SKOV-3 ovarian cancer cell lines as models for ovarian cancer in in vitro migration and invasion assays, we sought to understand the mechanisms associated with estrogen, progestin (medroxyprogesterone, MPA) and the PI3K/AKT pathway on metastasis.

\section{Materials and methods}

Cell lines and tissues. Human ovarian serous adenocarcinoma cell line SKOV-3 and clear cell carcinoma cell line ES-2 were obtained from ATCC and maintained in RPMI-1640 (Gibco BRL) containing 10\% fetal bovine serum (FBS) (Hyclone) in a humidified atmosphere with $5 \% \mathrm{CO}_{2}$ at $37^{\circ} \mathrm{C}$. We collected paraffin-embedded samples of 21 benign ovarian serous cystadenomas, 18 borderline ovarian serous borderline tumors, 17 primary ovarian clear cell carcinomas and 26 primary ovarian serous adenocarcinomas from patients who underwent surgery at the Gynecology and Obstetrics Hospital of Fudan University from January 2001 to December 2002. All tissues were obtained from patients after written consent using protocols and procedures approved by the Institutional Review Boards. Tumors were collected from patients who received no prior radiotherapy, chemotherapy, hormone therapy or immunotherapy and who did not present other types of cancer. Complete patient follow-up data was available for all the tissue samples used in the study.

Immunohistochemistry. We performed immunohistochemical staining for nm23-H1, total AKT and pAKT (phosphorSer473) protein on paraffin-embedded sections. Briefly, $4-\mu \mathrm{m}$ sections were deparaffinized, rehydrated and incubated with $0.3 \% \mathrm{H}_{2} \mathrm{O}_{2}$ to block endogenous peroxidase activity and heated in antigen-restoring solutions. The sections were incubated at $4^{\circ} \mathrm{C}$ overnight with anti-nm23-H1 monoclonal antibody (1:50, Santa Cruz Biotechnology Inc., Santa Cruz, CA), anti-AKT monoclonal antibody (1:500, Cell Signaling, 2967) or anti-pAKT (Ser473) monoclonal antibody (1:200, Cell Signaling). The sections were incubated with EnVision solution. The antigen-antibody complexes were visualized by $\mathrm{DAB}$ and counterstained with hematoxylin. Normal human colon mucosa sections provided the positive control for the binding of nm23-H1 antibody. Known positive ovarian epithelial cancers served as the positive controls for the binding of AKT and pAKT antibodies. The sections incubated with phosphate-buffered saline (PBS) instead of primary antibody served as the negative controls. Tumors were considered nm23-H1-, AKT- and pAKT-positive when $>5 \%$ of epithelial cells showed staining of the cytoplasm and membrane.

Wound healing assay. ES-2 and SKOV-3 cells were grown to $80 \%$ confluence in 12-well plates in Dulbecco's modified Eagle's medium (DMEM) containing $0.5 \%$ FBS. The cell monolayer was scratched using a sterilized $10-\mu 1$ pipette tip, washed and then subjected to $1 \times 10^{6} \mathrm{~mol} / 1$ 17- $\beta$ estrogen (Sigma) or $1 \times 10^{6} \mathrm{~mol} / 1 \mathrm{MPA}$ (Sigma). The migration of cells into the wound was imaged and monitored at 0,24 and $48 \mathrm{~h}$ after treatment. The images were analyzed in IMS Imaging Software provided by Shanghai Shenteng Information Technology Inc. Results were obtained from three individual experiments.

Cell migration assay. Migration of ES-2 and SKOV-3 cells through $8-\mu \mathrm{m}$ pores was assessed using a Transwell Cell Culture chamber (Corning Costar). The upper chambers were supplemented with DMEM media containing 0.5\% FBS while the lower chambers were supplemented with complete media. Cells $(100 \mu \mathrm{l})$ at the density of $1 \times 10^{5} / \mathrm{ml}$ were seeded onto the upper surface of the culture insert with $1 \times 10^{6} \mathrm{~mol} / \mathrm{l}$ $17-\beta$ estrogen or MPA with or without AKT siRNA. Nonsense siRNA served as the control. After $12 \mathrm{~h}$, the cells on the upper surface of the membrane insert were removed with a cotton swab, and the migrated cells which had migrated to the underside of the membrane insert were fixed with methanol and stained with hematoxylin. The number of cells was counted in five random high-power fields (HPFs) using a light microscope. For each experimental condition, the assay was performed in quadruplicate. The assay was repeated three times.

Treatment of cell lines with estrogen, progestin, PIK3 kinase inhibitor and MAPK inhibitor. To study the effect of hormones on the expression of nm23-H1, ES-2 and SKOV-3 cells were pretreated with RPMI-1640 media without phenol red containing $10 \%$ active carbon-treated FBS for $72 \mathrm{~h}$. In dosedependent experiments to investigate $\mathrm{nm} 23-\mathrm{H} 1$ expression after hormone treatment, cells were treated with serial concentrations $\left(10^{-8}, 10^{-7}, 10^{-6} \mathrm{~mol} / \mathrm{l}\right)$ of $17-\beta$ estrogen or MPA and harvested $24 \mathrm{~h}$ later. In the time-dependent experiments, cells were treated with $10^{-6} \mathrm{~mol} / 1$ 17- $\beta$ estrogen or MPA for 24,48 and $72 \mathrm{~h}$, respectively. To study the effect of hormones on AKT and pAKT expression, cells were treated with serial concentrations $\left(10^{-9}, 10^{-8}, 10^{-7}, 10^{-6}, 10^{-5} \mathrm{~mol} / \mathrm{l}\right)$ of $17-\beta$ estrogen or MPA for $1 \mathrm{~h}$ or treated with $10^{-6} \mathrm{~mol} / 1 \mathrm{1}-\beta$ estrogen or MPA for different time periods (30 min and 1, 2, $4,8,16$ and $24 \mathrm{~h})$.

To study the combined effect of hormones and pharmacological inhibitors, ES-2 and SKOV-3 cells were treated with $10^{-6} \mathrm{~mol} / 1 \mathrm{l}-\beta$ estrogen or $10^{-6} \mathrm{~mol} / \mathrm{l} \mathrm{MPA}$ and $25 \mu \mathrm{mol} / \mathrm{l}$ PIK3 kinase inhibitor LY294002 or MAPK inhibitor PD98059 (both from Calbiochem, CA) for $1 \mathrm{~h}$. DMSO served as the negative control. nm23-H1, AKT and pAKT protein expression was detected by Western blot analysis.

siRNA assay. ES-2 and SKOV-3 cells at $60 \%$ confluency were transfected with AKT siRNA (Cell Signaling) at the concentration of $4 \%$ per sample by Oligofectamine reagent (Invitrogen) and in accordance with the manufacturer's 
instructions. Nonsense siRNA served as the negative control. After $24 \mathrm{~h}$, transfection efficiency was measured by fluorescence microscopy, and AKT expression was detected by Western blotting. For combination treatment (ie., cells transfected with AKT siRNA for $24 \mathrm{~h}$ and treated with estrogen or MPA for $24 \mathrm{~h}$ ) $\mathrm{nm} 23-\mathrm{H} 1$ protein expression was measured using Western blot analysis.

Western blot analysis. Cells with and without treatment were washed with cold PBS and harvested using RIPA buffer containing protease inhibitor cocktail. Cell lysates were incubated on ice for $30 \mathrm{~min}$. After centrifugation at 14,000 rpm for $10 \mathrm{~min}$ at $4^{\circ} \mathrm{C}$, protein concentration was determined using a Bio-Rad protein assay (Bio-Rad, Hercules, CA). Proteins were separated on $10 \%$ SDS-PAGE and transferred to a PVDF membrane (Millipore, Bedford, MA). The membranes were blocked by $5 \%$ skimmed milk, and the proteins of interest were detected by anti-nm23-H1 monoclonal antibody (1:3000), anti-AKT monoclonal antibody (1:1000) and antipAKT (Ser 473) monoclonal antibody (1:1000), respectively. The blots were then stripped and blotted with a primary antibody against GAPDH (1:3000, Sigma) for equal loading control.

Statistical analysis. Statistical analyses were performed using SPSS10.0 Software. Difference between clinical groups was examined with the Chi-square test. The Student's t-test was used to compare means in different groups. Assuming normal distribution, $\mathrm{p}<0.05$ was determined to be indicative of a significant difference and a $95 \%$ confidence interval; that is, the differences between the means were true.

\section{Results}

nm23-H1, AKT, pAKT expression and clinicopathological features. We performed immunohistochemical staining and measured nm23-H1, AKT, pAKT expression in 21 benign ovarian serous cystadenomas, 18 borderline ovarian serous cystadenomas, 17 primary ovarian clear cell carcinomas and 26 primary ovarian serous adenocarcinomas. There were no differences in patient age among the four groups. There were no differences in tumor stage, grade, lymph node status and survival between patients of clear cell carcinomas and serous adenocarcinomas. We found that $\mathrm{nm} 23-\mathrm{H} 1$ was positively expressed in $33.3 \%$ of patients with benign cystadenoma, $66.7 \%$ of patients with borderline cystadenoma, $57.7 \%$ of patients with serous adenocarcinoma and $11.8 \%$ of cases with clear cell carcinoma. On the other hand, AKT was rarely expressed in benign and borderline cystadenomas (4.8 and $27.8 \%$ respectively) and frequently expressed in serous adenocarcinomas $(80.8 \%)$. Similarly, positive expression of pAKT in benign, borderline serous cystadenomas and serous adenocarcinomas was 19, 22.2 and $76.9 \%$ respectively. For clear cell carcinoma, nm23-H1, AKT and pAKT expression was $11.8,11.8$ and $94.1 \%$ respectively (Fig. 1B).

We examined the association between nm23-H1, AKT and $\mathrm{pAKT}$ protein expression and clinicopathological features of the patients with primary ovarian serous carcinoma and clear cell carcinoma. As shown in Table I, we found that $\mathrm{nm} 23-\mathrm{H} 1, \mathrm{AKT}$ and $\mathrm{pAKT}$ expression was negatively associated with age at diagnosis and clinical stage of serous adenocarcinoma patients. nm23-H1 was more frequently expressed in serous adenocarcinoma patients with tumor grade 1 than grade 2 or $3(\mathrm{p}<0.01)$, whereas AKT and pAKT were more frequently expressed in serous adenocarcinoma patients with tumor grade 2 or 3 than grade 1 $(\mathrm{p}<0.05)$. We also found $\mathrm{nm} 23-\mathrm{H} 1$ expression was positively associated with 5-year survival whereas AKT and pAKT expression were negatively associated with 5-year survival. $\mathrm{nm} 23-\mathrm{H} 1$ expression was negatively associated with lymph node metastasis $(\mathrm{p}<0.01)$ (Table I).

There was no correlation between nm23-H1, AKT and pAKT expression and patient age, tumor stage, 5-year survival and status of lymph node metastasis in ovarian clear cell carcinoma (Table II).

Effect of estrogen and progestin on migration and invasion of ES-2 and SKOV-3 cells. In the wound healing assay, 17- 3 estrogen significantly stimulated the migration of both ES-2 and SKOV-3 cells after 24 and $48 \mathrm{~h}$ of treatment (Fig. 2A and B). The lesion distance of ES-2 cells decreased from $0.83 \pm 0.02 \mathrm{~mm}$ in the control cells to $0.66 \pm 0.06 \mathrm{~mm}$ in the estrogen-treated cells $(\mathrm{p}=0.014)$ after $48 \mathrm{~h}$ of treatment. In the SKOV-3 cells which were treated for $48 \mathrm{~h}$, the lesion distance was $0.25 \pm 0.02 \mathrm{~mm}$ in the control group and $0.18 \pm 0.02 \mathrm{~mm}$ in the estrogen group $(\mathrm{p}=0.015)$. After $1 \times 10^{-6} \mathrm{~mol} / \mathrm{l} \mathrm{MPA}$ treatment for 24 and $48 \mathrm{~h}$, cell migration was inhibited in both ES-2 and SKOV-3 cells. The lesion distance of ES-2 cells increased from $0.66 \pm 0.06 \mathrm{~mm}$ in the control cells to $0.93 \pm 0.02 \mathrm{~mm}$ in 48-h MPA-treated cells $(\mathrm{p}=0.001)$. In the SKOV-3 cells, the lesion distance was $0.18 \pm 0.02 \mathrm{~mm}$ in the control group and $0.36 \pm 0.03 \mathrm{~mm}$ in the 48 -h MPA group $(\mathrm{p}=0.015)$ (Fig. 2C and D).

In the cell migration assay, both ES-2 and SKOV-3 cells migrating through the porous membrane transwell cell culture chamber were also stimulated by estrogen and suppressed by progestin, respectively. The average ES-2 cell count crossing the matrigel-coated membrane in 5 HPFs was $92.3 \pm 15.6$ in the control group, $118.7 \pm 12.7$ in the estrogen group $(\mathrm{p}=0.015)$ and $77.8 \pm 7.2$ in the MPA group $(\mathrm{p}=0.006)$. The average SKOV-3 cell count was $122.7 \pm 10.2$ in the control group, $166.7 \pm 7.4$ in the estrogen group $(p<0.001)$ and $90.3 \pm 14.7$ in the MPA group ( $\mathrm{p}=0.011)$ (Fig. $3 \mathrm{~A}$ and $\mathrm{B}$ ).

Estrogen and progestin regulate the expression of $n m 23-H 1$, $A K T$ and $p A K T$ in ovarian cancer cells. According to the effect of estrogen and progestin on migration and invasion of ovarian cancer cells, we investigated the expression of $\mathrm{nm} 23-\mathrm{H} 1$, the metastasis-related protein. Western blot analysis showed that estrogen reduced $\mathrm{nm} 23-\mathrm{H} 1$ expression in ES-2 and SKOV-3 cells in a dose- and time-dependent manner. Even a low dose of $17-\beta$ estrogen $\left(10^{-8} \mathrm{~mol} / \mathrm{l}\right)$ reduced $\mathrm{nm} 23$ H1 expression, and this suppressive effect reached a peak at the dose of $10^{-6} \mathrm{~mol} / \mathrm{l}(\mathrm{p}<0.05)$. Regarding the time course, nm23-H1 expression was slightly reduced after a 24-h treatment, and this reduction reached a peak after a $72-\mathrm{h}$ treatment at the dose of $10^{-6} \mathrm{~mol} / 1$ 17- $\beta$ estrogen. As shown in Fig. 4A, MPA increased nm23-H1 expression in ES-2 and SKOV-3 cells also in a dose- and time-dependent manner. 

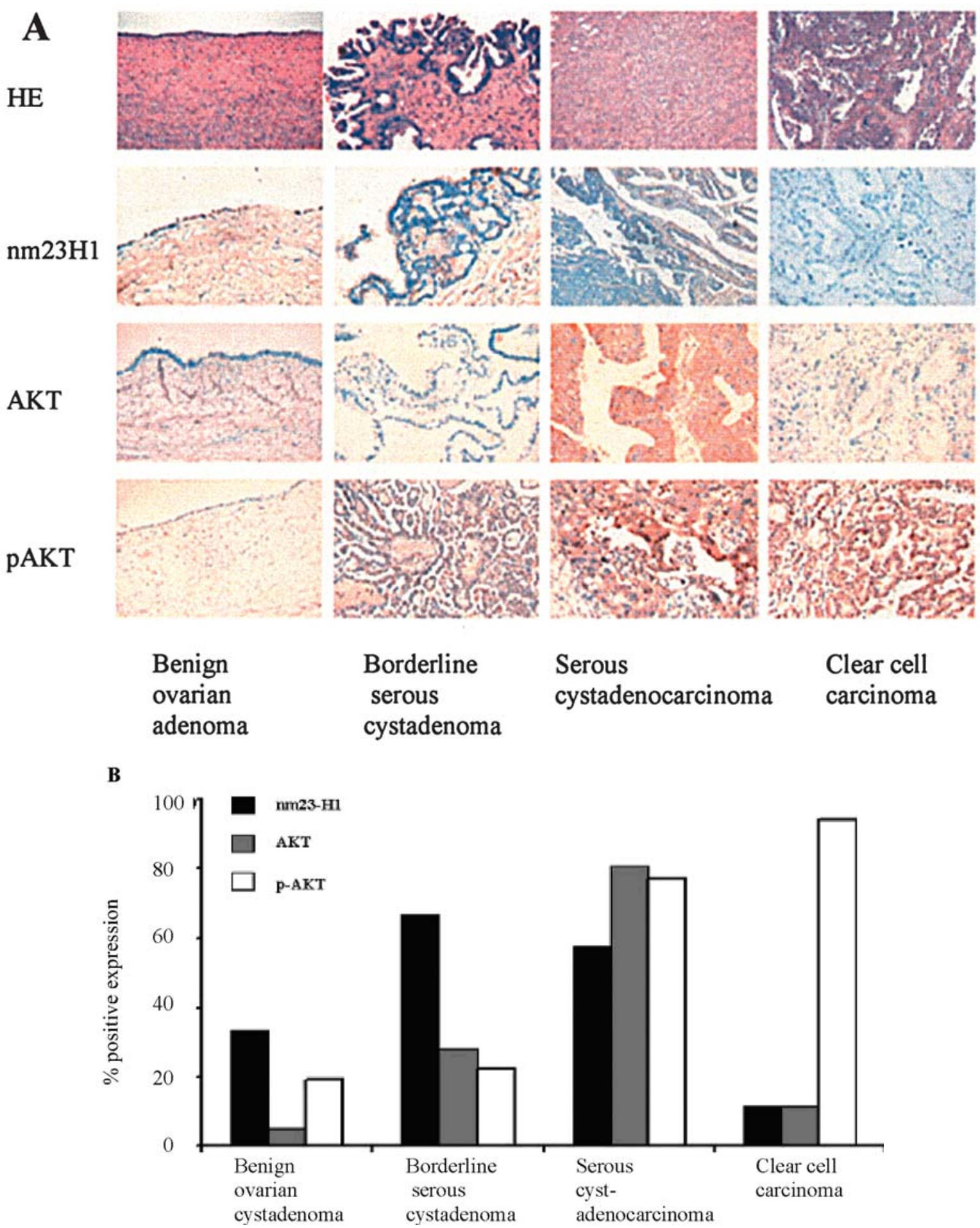

Figure 1. nm23-H1, AKT, pAKT expression in ovarian benign, borderline serous cystadenomas, ovarian clear cell carcinomas and serous adenocarcinomas. (A) From top to bottom: morphologic features of four types of tumors (H\&E, x200). (B) Percentage of positive nm23-H1, AKT and pAKT expression in the four types of tumors. Tumors were considered nm23-H1-, AKT- and pAKT-positive when $>5 \%$ of epithelial cells showed staining of the cytoplasm and membrane.

To determine whether the PIK3/AKT pathway was involved in the effect of estrogen and progestin on nm23-H1 expression, we further examined AKT and pAKT expression after hormone treatment. pAKT expression was increased significantly after a 1-h treatment with 17- $\beta$ estrogen, starting at the low dose of $10^{-8} \mathrm{~mol} / \mathrm{l}$ in ES-2 and SKOV-3 cells. In regards to the time point, $10^{-6} \mathrm{~mol} / 1 \mathrm{l} 17-\beta$ estrogen increased pAKT expression as early as $30 \mathrm{~min}$ after treatment. This increase reached a peak at $30 \mathrm{~min}$ and $2 \mathrm{~h}$ in ES-2 and SKOV-3 cells, respectively. MPA induced a suppressive effect on pAKT expression in both cell lines. Neither 17- $\beta$ estrogen nor MPA affected AKT expression (Fig. 4B). 
Table I. nm23-H1, AKT, pAKT expression and clinical characteristics of serous adenocarcinoma patients.

\begin{tabular}{|c|c|c|c|c|c|c|c|}
\hline & \multicolumn{7}{|c|}{ No. of patients } \\
\hline & \multicolumn{2}{|c|}{$\mathrm{nm} 23-\mathrm{H} 1$} & \multicolumn{2}{|c|}{ AKT } & \multicolumn{3}{|c|}{$\mathrm{pAKT}$} \\
\hline & + & - & + & - & + & & - \\
\hline \multicolumn{8}{|c|}{ Patient age (years) } \\
\hline$>50$ & 9 & 6 & 12 & 3 & 12 & & 3 \\
\hline$\leq 50$ & 6 & 5 & 9 & 2 & 8 & & 3 \\
\hline p-value & \multicolumn{2}{|c|}{1.000} & \multicolumn{2}{|c|}{1.000} & \multicolumn{3}{|c|}{0.670} \\
\hline \multicolumn{8}{|c|}{ Tumor stage } \\
\hline I or II & 9 & 7 & 13 & 3 & 12 & & 4 \\
\hline III or IV & 6 & 4 & 8 & 2 & 8 & & 2 \\
\hline $\mathrm{p}$-value & \multicolumn{2}{|c|}{1.000} & \multicolumn{2}{|c|}{1.000} & \multicolumn{3}{|c|}{1.000} \\
\hline \multicolumn{8}{|c|}{ Tumor grade } \\
\hline 1 & 6 & 0 & 2 & 4 & 2 & & 4 \\
\hline 2 or 3 & 9 & 11 & 19 & 1 & 18 & & 2 \\
\hline $\mathrm{p}$-value & \multicolumn{2}{|c|}{0.024} & \multicolumn{2}{|c|}{0.005} & \multicolumn{3}{|c|}{0.013} \\
\hline \multicolumn{8}{|c|}{ Patient survival } \\
\hline$<5$ years & 8 & 11 & 18 & 1 & 17 & & 2 \\
\hline$\geq 5$ years & 7 & 0 & 3 & 4 & 3 & & 4 \\
\hline $\mathrm{p}$-value & \multicolumn{2}{|c|}{0.010} & \multicolumn{2}{|c|}{0.010} & \multicolumn{3}{|c|}{0.028} \\
\hline \multicolumn{8}{|c|}{ Lymph node metastasis } \\
\hline Positive & 1 & 6 & 7 & 0 & 7 & & 0 \\
\hline Negative & 14 & 5 & 14 & 5 & 14 & & 5 \\
\hline $\mathrm{p}$-value & \multicolumn{2}{|c|}{0.021} & \multicolumn{2}{|c|}{0.278} & \multicolumn{3}{|c|}{0.278} \\
\hline
\end{tabular}

Table II. nm23-H1, AKT, pAKT expression and clinical characteristics of clear cell adenocarcinoma patients.

\begin{tabular}{|c|c|c|c|c|c|c|c|}
\hline & \multicolumn{7}{|c|}{ No. of patients } \\
\hline & \multicolumn{2}{|c|}{$\mathrm{nm} 23-\mathrm{H} 1$} & \multicolumn{2}{|c|}{ AKT } & \multicolumn{3}{|c|}{ pAKT } \\
\hline & + & - & + & - & + & & - \\
\hline \multicolumn{8}{|c|}{ Patient age (years) } \\
\hline$>50$ & 0 & 0 & 0 & 0 & 9 & & 1 \\
\hline$\leq 50$ & 0 & 0 & 0 & 0 & 7 & & 0 \\
\hline p-value & \multicolumn{2}{|c|}{-} & \multicolumn{2}{|c|}{-} & \multicolumn{3}{|c|}{0.390} \\
\hline \multicolumn{8}{|c|}{ Tumor stage } \\
\hline I or II & 2 & 11 & 0 & 13 & 12 & & 1 \\
\hline III or IV & 0 & 4 & 2 & 2 & 4 & & 0 \\
\hline p-value & \multicolumn{2}{|c|}{1.000} & \multicolumn{2}{|c|}{0.044} & \multicolumn{3}{|c|}{1.000} \\
\hline \multicolumn{8}{|c|}{ Patient survival } \\
\hline$<5$ years & 0 & 8 & 2 & 6 & 0 & & 8 \\
\hline$\geq 5$ years & 2 & 7 & 0 & 9 & 8 & & 1 \\
\hline p-value & \multicolumn{2}{|c|}{0.470} & \multicolumn{2}{|c|}{0.206} & \multicolumn{3}{|c|}{0.000} \\
\hline \multicolumn{8}{|c|}{ Lymph node metastasis } \\
\hline Positive & 0 & 2 & 2 & 0 & 2 & & 0 \\
\hline Negative & 2 & 13 & 0 & 5 & 14 & & 1 \\
\hline p-value & \multicolumn{2}{|c|}{1.000} & \multicolumn{2}{|c|}{0.048} & \multicolumn{3}{|c|}{1.000} \\
\hline
\end{tabular}




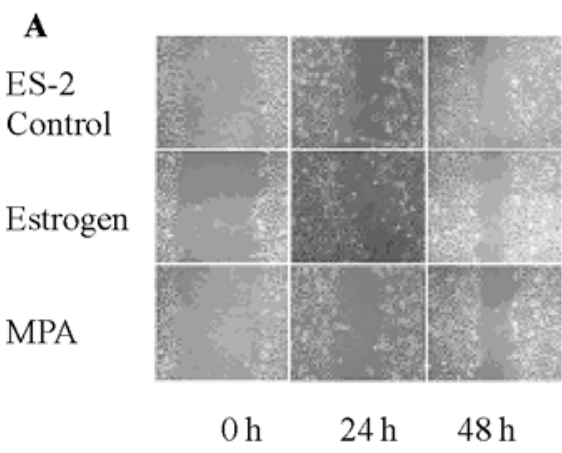

C

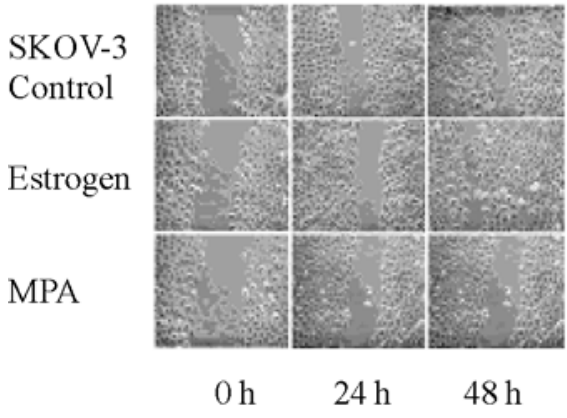

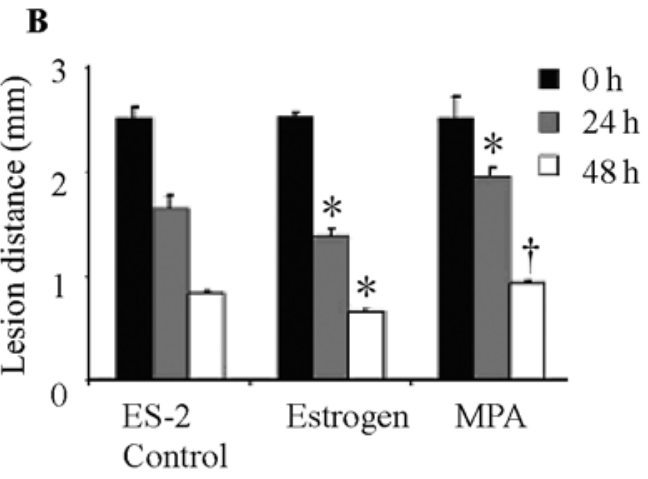

D

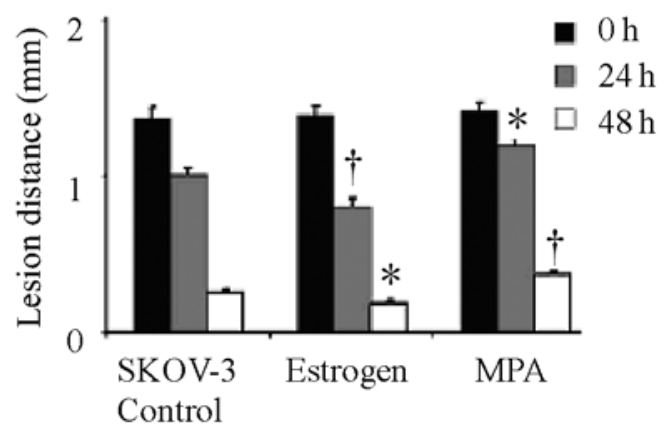

Figure 2. Estrogen and progestin differentially altered ES-2 and SKOV-3 cell migration in a wound healing assay. A monolayer of ES-2 (A) and SKOV-3 (C) cells were scratched with a sterile pipette tip. Repair of lesion by cell migration without or with $1 \times 10^{-6}$ mol/1 $17-\beta$ estrogen or $1 \times 10^{-6}$ mol/1 MPA were photographed for the initial wounding $(0 \mathrm{~h})$ and at 24 and $48 \mathrm{~h}$ post-wounding. The distances of the lesions of ES-2 (B)and SKOV-3 (D) cells were quantified at the given times. Data represent the mean $\pm \mathrm{SE}$ and were obtained from three independent experiments. ${ }^{*} \mathrm{p}<0.05$, ${ }^{\dagger} \mathrm{P}<0.01$ compared with untreated cells at the given time points; $0 \mathrm{~h}$ (black bar), $24 \mathrm{~h}$ (light grey bar) and $48 \mathrm{~h}$ (white bar).
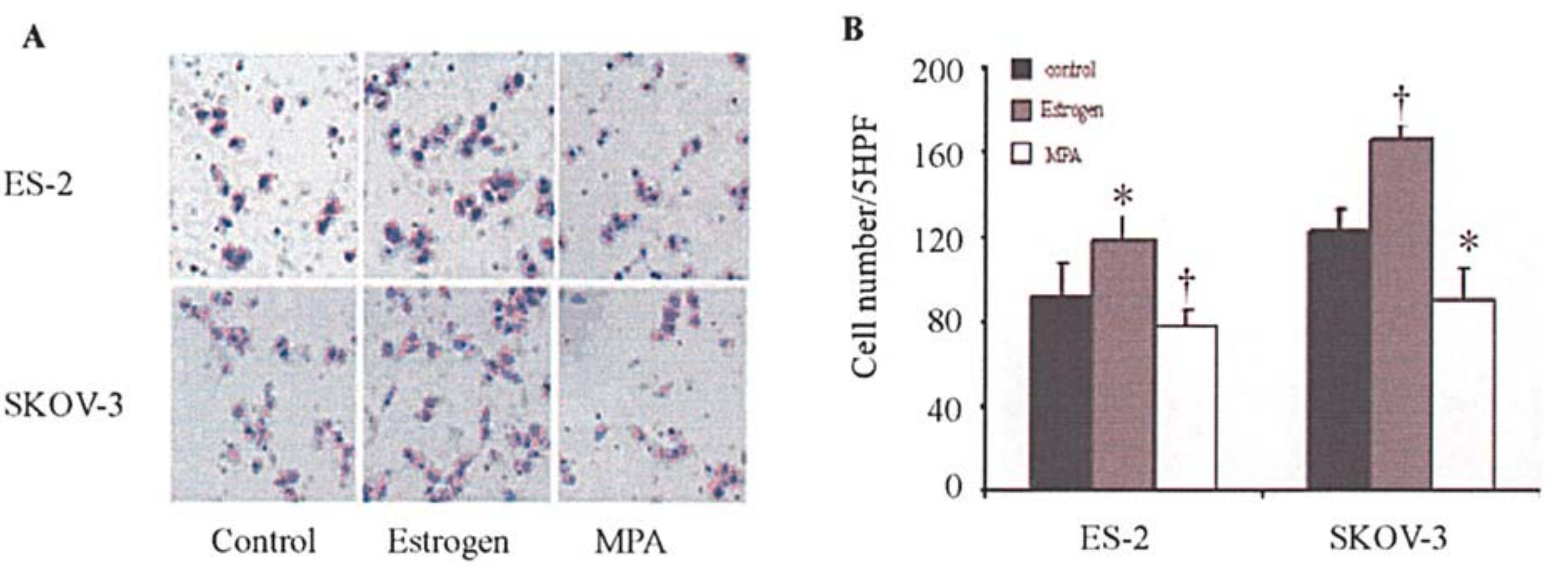

Figure 3. Estrogen stimulated the migration of ES-2 and SKOV-3 cells in the cell migration assay; whereas MPA had an inhibitory effect. (A) Images of ES-2 and SKOV-3 cells migrating through the porous membrane in the control, $1 \times 10^{-6} \mathrm{~mol} / 1$ estrogen and $1 \times 10^{-6} \mathrm{~mol} / 1 \mathrm{MPA}$ groups. (B) Average cell count of invaded cells per 5HPFs in three groups. The data represent the results obtained from at least three independent experiments. ${ }^{*} \mathrm{p}<0.05$, ${ }^{\dagger} \mathrm{P}<0.01$ compared with untreated cells.

Modulation of AKT interfered with the effect of estrogen and progestin on ES-2 and SKOV-3 cell migration and nm23-H1 expression. Estrogen induced pAKT expression earlier than $\mathrm{nm} 23-\mathrm{H} 1$, and progestin exerted the opposite effect. To define the function of AKT, we used AKT siRNA to specifically down-regulate endogenous AKT expression, and we reassessed cell migration and $\mathrm{nm} 23-\mathrm{H} 1$ expression in ES-2 and SKOV-3 cells. As shown in Fig. 5A, the transient transfection of AKT siRNA into ES-2 and SKOV-3 cells significantly reduced AKT expression by $50-60 \%(\mathrm{p}<0.01)$ compared with nonsense siRNA transfection.

Despite the incomplete siRNA silencing of AKT expression, in the cell migration assay, the number of cells that invaded to the lower surfaces of the membranes in the estrogen combined with AKT siRNA group was significantly less than the 17- $\beta$ estrogen plus nonsense siRNA control 

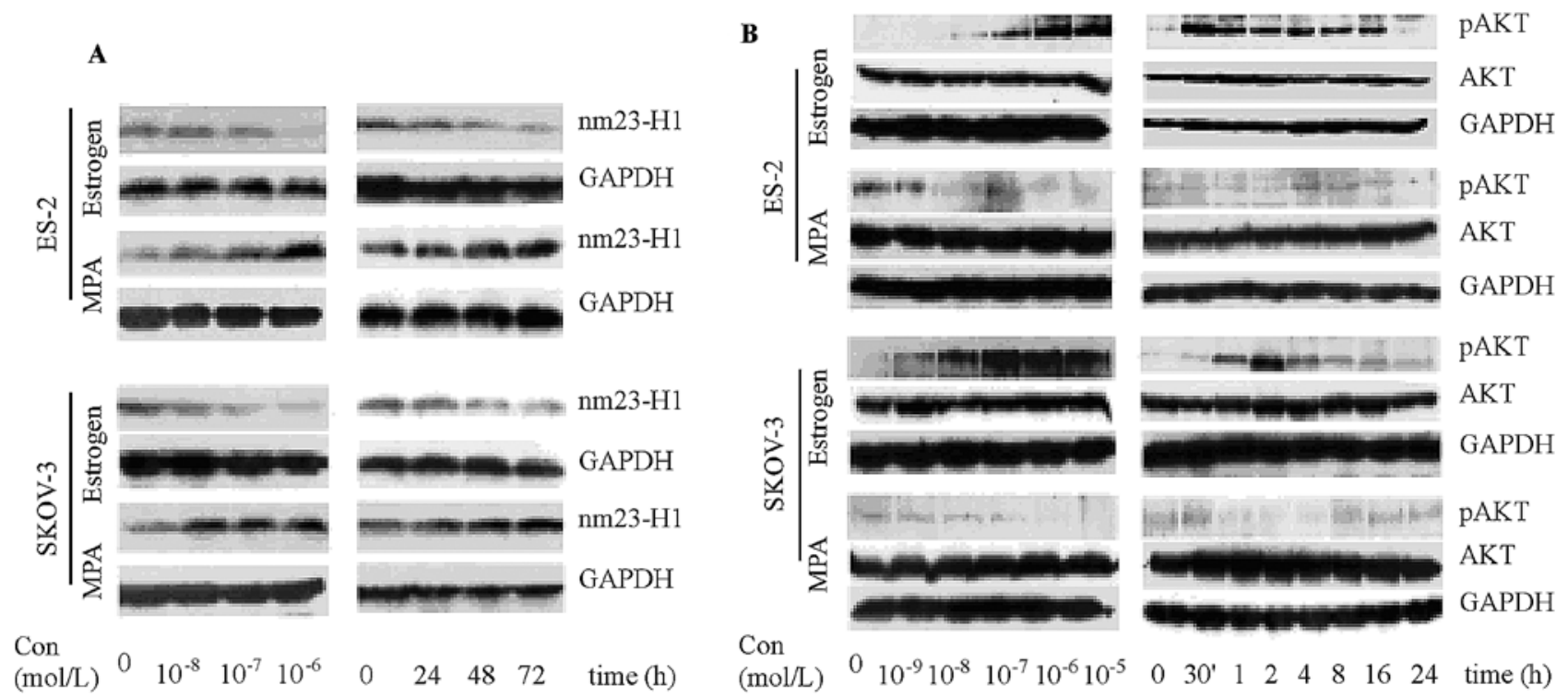

Figure 4. Estrogen suppressed nm23-H1 and increased AKT and pAKT expression in ovarian cancer cells, whereas progestin had an opposite effect. (A) 17-ß estrogen and MPA differentially altered the expression of nm23-H1 in a dose- and time-dependent manner in ES-2 and SKOV-3 cells. nm23-H1 protein levels were determined by Western blot analysis with anti-nm23-H1 antibody. GAPDH was used as the loading control. (B). 17-ß estrogen and MPA altered pAKT expression in a dose- and time-dependent manner in both ES-2 and SKOV-3 cells. Total AKT protein level was not altered. Protein level was determined by Western blot analysis with active AKT (phosphor-Ser473) and total AKT. GAPDH was used as the loading control.

5A
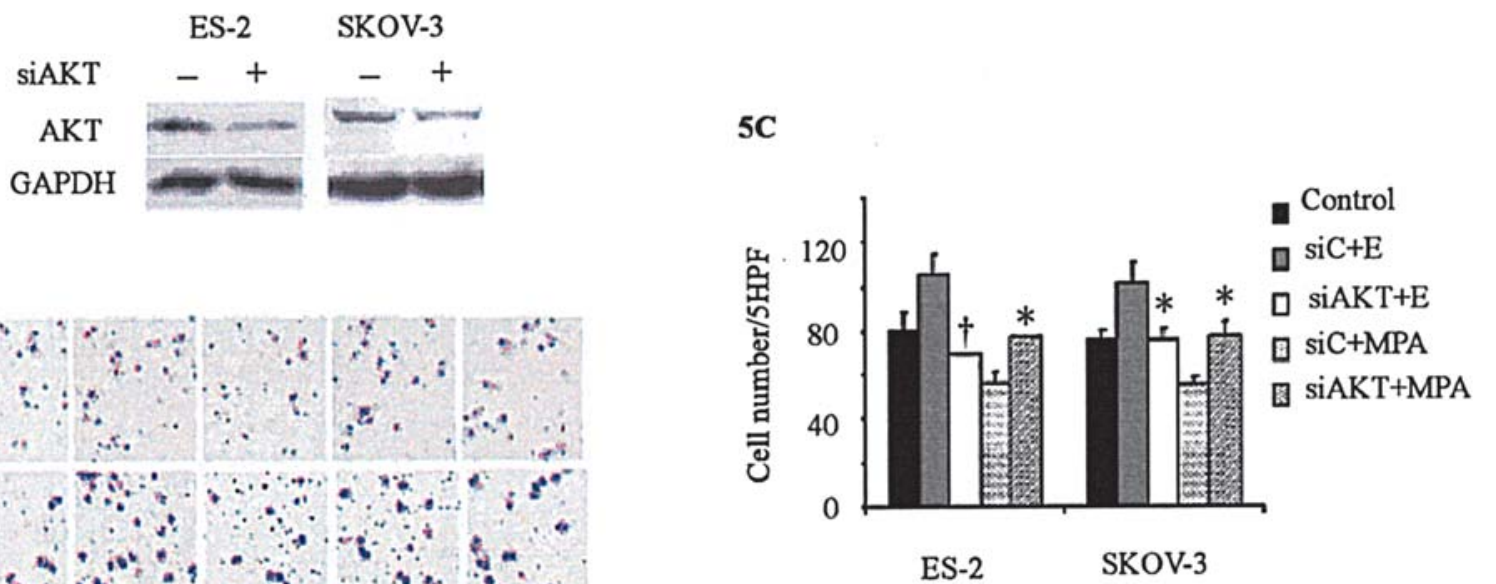

Figure 5. Modulation of AKT in ES-2 and SKOV-3 cells affected estrogen- and MPA-induced cell migration. (A) Cells were transiently transfected with AKT siRNA. Total AKT expression was detected by Western blot analysis, normalized to GAPDH as a total protein loading control. (B) Images of ES-2 and SKOV-3 cells migrating through the porous membrane in the transfection reagent alone control (Control), estrogen or MPA plus nonsense siRNA groups ( $\mathrm{siC}+\mathrm{E}$ or siC+MPA) and estrogen or MPA plus Akt siRNA (siAKT+E or siAKT+MPA) treatment groups. (C) The number of migrating cells was measured in each group. The data represent the results from at least three independent experiments. ${ }^{*} \mathrm{p}<0.05$, ${ }^{\dagger} \mathrm{P}<0.01$ compared with estrogen or MPA plus nonsense siRNA group.

group. On the other hand, combined with AKT siRNA treatment, MPA inhibition of cell invasion was enhanced (Fig. 5B and C). These results suggest that the PIK3/AKT pathway might be involved in the regulation of estrogen and progestin in cell invasion.

Since after estrogen treatment, pAKT protein upregulation occurred earlier than $\mathrm{nm} 23-\mathrm{H} 1$ protein downregulation, we speculated that using siAKT to knock-down AKT expression could modify the effect of estrogen on
nm23-H1 expression. As expected, upon treatment with AKT siRNA, the suppressive effect of estrogen on nm23-H1 was restored $(\mathrm{p}<0.05)$ compared to treatment with nonsense siRNA in ES-2 and SKOV-3 cells. Likewise, AKT siRNA treatment decreased the up-regulation of $n m 23-\mathrm{H} 1$ by progestin ( $\mathrm{p}>0.05$ ) (Fig. 6A).

In order to further confirm the involvement of the PIK3/ AKT pathway in the hormone regulation of $\mathrm{nm} 23-\mathrm{H} 1$ expression, we treated ES-2 and SKOV-3 cells with the 
A

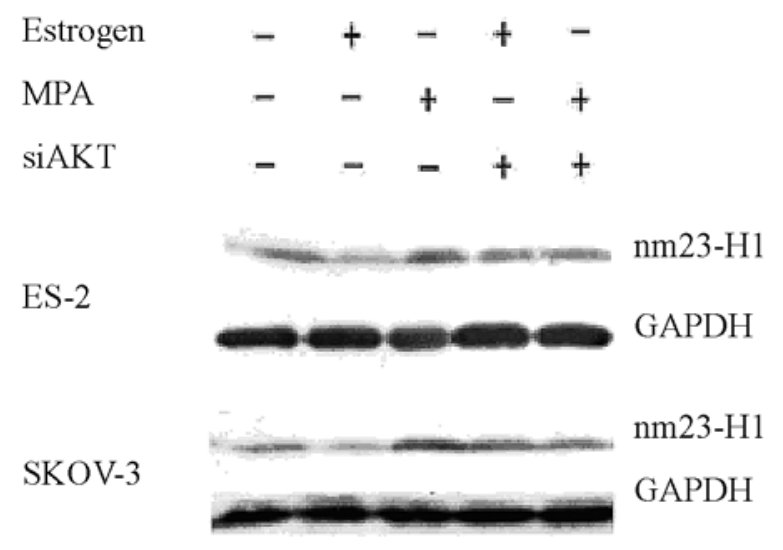

B

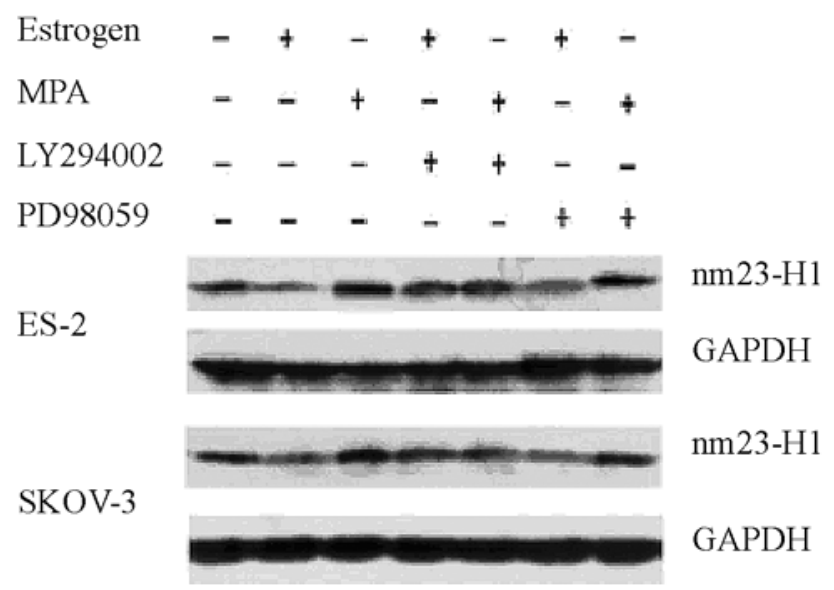

Figure 6. The PIK3/AKT pathway is involved in hormone regulation of nm23-H1 expression. (A) nm23-H1 protein expression was assessed by Western blot analysis in ES-2 and SKOV-3 cells treated with 17-ß estrogen, MPA, siAKT combined with 17- 3 estrogen or MPA. GAPDH served as the loading control. (B) nm23-H1 protein expression was assessed by Western blot analysis in ES-2 and SKOV-3 cells treated with 17-ß estrogen, MPA, LY294002, PD98059 only or both. GAPDH served as the loading control.

PIK3/AKT inhibitor LY294002 or the MAPK inhibitor PD980059 combined with estrogen or MPA, and then reassessed nm23-H1 expression. As expected, treatment with LY294002 decreased the down-regulation of nm23-H1 by estrogen in both ES-2 and SKOV-3 cells. Likewise, LY294002 treatment decreased the up-regulation of $\mathrm{nm} 23-\mathrm{H} 1$ expression by MPA. Treatment with PD980059 had no effect on the hormone-induced change of $\mathrm{nm} 23-\mathrm{H} 1$ expression. These results further support the association between the PIK3/AKT pathway and the influence of hormones on cell metastasis.

\section{Discussion}

Migration of cancer cells from a primary tumor to a site of potential metastasis depends on a series of complex biological steps, regulated by multiple genes. It is well known that advanced ovarian cancer is characterized by a high frequency of metastasis to lymph nodes and invasive growth into multiple organs due to peritoneal dissemination. The 5-year survival rate of patients with advanced ovarian carcinoma is only $30 \%$ compared to $60-70 \%$ of early stage patients. Thus, it is urgent to develop innovative approaches for the treatment of ovarian cancer. Defining genetic aberrations and their underlying molecular changes involved in the process of metastasis can aid in the treatment of ovarian cancers.

In this study, we evaluated anti-metastatic protein nm23$\mathrm{H} 1, \mathrm{AKT}$ as well as pAKT in ovarian serous tumors and clear cell carcinomas. nm23-H1, a metastasis suppressor gene, is expressed in normal cells and participates in cellular message transduction and in maintaining normal physiological function. Down-regulation of nm23-H1 was reported to induce meiosis and spindle rearrangement, alter the cell skeleton, facilitate cell movement, and be involved in cell migration, invasion and metastasis. Our results showed that $\mathrm{nm} 23-\mathrm{H} 1$ was expressed in $33.3 \%$ of patients with benign serous cystadenomas, $66.7 \%$ of patients with borderline serous tumors and $57.7 \%$ of patients with serous adenocarcinomas. nm23-H1 expression was negatively related to clinical tumor stage, grade and lymph node metastasis and positively related to survival. Our results suggest that increased levels of nm23$\mathrm{H} 1$ are associated with a favorable prognosis for ovarian serous adenocarcinomas. In benign tumors, highly expressed $\mathrm{nm} 23-\mathrm{H} 1$ inhibited cell proliferation in contrast with that in malignant tumors. Decreased nm23-H1 expression altered cell behavior such as proliferation and differentiation, suggesting that the loss of nm23-H1 expression is involved in the process of benign to malignant transition $(16,17)$. Furthermore, a low level of nm23-H1 was associated with high invasiveness of clear cell carcinoma, implicating that nm23-H1 downregulation tends to promote development, invasion and metastasis in clear cell carcinomas (18). In support of our results, Brustmann and Naude (19) reported that nm23-H1 was not or slightly expressed in normal or hyperplasia endometrium and expressed in $52.7 \%$ of endometrial carcinomas relative to tumor grade. $\mathrm{nm} 23-\mathrm{H} 1$ expression is not only an indicator of metastasis but also a marker for evaluating metastasis, recurrence and prognosis.

The PI3K/AKT cell-signaling pathway is implicated in cell migration and invasion (19-21). AKT has been shown to be amplified or overexpressed in ovarian cancer, implying that it has a role in ovarian carcinogenesis (9-11). In the present study, we found that the levels of AKT and pAKT increased from benign, borderline to malignant serous tumors. AKT and pAKT expression was associated with tumor grade, lymph node involvement and 5-year survival in ovarian serous adenocarcinomas. Tumors with positive AKT and pAKT expression are more aggressive and are associated with a poor patient prognosis. These results suggest that AKT and pAKT expression is, not only an indicator of cell differentiation, but also a marker of patient prognosis.

Estrogen has been reported to play a role in ovarian cancer carcinogenesis. Our study verified that estrogen stimulates cell migration and down-regulates metastasis suppressor gene nm23-H1 expression, whereas progestin inhibits cell migration and induces $\mathrm{nm} 23-\mathrm{H} 1$ expression. Therefore, it is imperative to understand the hormone regulation of $\mathrm{nm} 23-\mathrm{H} 1$ expression 
through signal pathways involved in migration and invasion of cancer cells which contribute to the progression and mortality of ovarian cancer.

The PI3K/AKT pathway is involved in many cellular processes, including proliferation, survival, apoptosis, migration, invasion and cytoskeletal rearrangements. Whitley et al (22) reported that insulin and IGF-1 are potential initiators of the PI3K/AKT pathway, which can alter the plasminogen activator system which subsequently influences SKOV-3 cell migration and invasion. The findings presented in this study dissected the importance of this pathway using pharmacological inhibitors, target deletion of AKT in estrogen- or progestin-altered cell migration and invasion with respect to the regulation of $\mathrm{nm} 23-\mathrm{H} 1$ expression. Estrogen, similar to a growth factor, stimulated the intracellular signal transduction pathway in a dose- and time-dependent manner. The phosphorylated AKT was induced by estrogen treatment as early as $30 \mathrm{~min}$. Subsequently, nm23-H1 expression was reduced slightly after estrogen treatment for $24 \mathrm{~h}$ and was reduced greatly after treatment for $72 \mathrm{~h}$. Knock-down of AKT expression by siRNA attenuated the stimulating effect of estrogen on cell invasion and migration. Furthermore, the decrease in nm23H1 expression by estrogen was also blocked by AKT siRNA interference. Similarly, treatment with PI3K inhibitor LY294002 also antagonized the decrease in $\mathrm{nm} 23-\mathrm{H} 1$ expression induced by estrogen. In contrast, the decrease in cell migration and invasion that accompanied the increase in $\mathrm{nm} 23-\mathrm{H} 1$ levels induced by progestin was enhanced by AKT siRNA interference.

The 'traditional' function of estrogen via ER has been well documented. However, the 'non-traditional' functions for estrogen, including cell migration and invasion, via the cell signaling pathway have not been investigated. Our results have expanded our understanding of the relationship between hormone induction of cell metastasis and the PI3K/AKT signaling pathway. Estrogen can down-regulate nm23-H1 expression through activating the PI3K/AKT pathway. Likewise, progestin, in conjunction with the inhibition of the PI3K/AKT pathway can effectively decrease cell migration and invasion by up-regulating nm23-H1 expression. In contrast, the MAPK inhibitor did not affect hormone induction of nm23-H1 expression and cell invasion, suggesting that the extracellular signal-regulated kinase (mitogen-activated protein kinase, MAPK) pathway was not involved in sex hormone regulation of $\mathrm{nm} 23-\mathrm{H} 1$ expression and cell invasion.

\section{References}

1. Ho C-M, Chien T-Y, Shih B-Y and Huang S-H: Evaluation of complete surgical staging with pelvic and para-aortic lymphadenectomy and paclitaxel plus carboplatin chemotherapy for improvement of survival in stage I ovarian clear cell carcinoma. Gynecol Oncol 88: 394-399, 2003.

2. Sugiyama T, Kamura T, Kigawa J, Terakawa N, Kikuchi Y, Kita T, Suzuki M, Sato I and Taguchi K: Clinical characteristics of clear cell carcinoma of the ovary: a distinct histologic type with poor prognosis and resistance to platinum-based chemotherapy. Cancer 88: 2584-2589, 2000.

3. Jensen JT and Speroff L: Health benefits of oral contraceptives. Obstet Gynecol Clin North Am 27: 705-721, 2000.

4. Vessey MP and Painter R: Endometrial and ovarian cancer and oral contraceptives - findings in a large cohort study. Br J Cancer 71: 1340-1342, 1995.
5. Schildkraut JM, Calingaert B, Marchbanks PA, Moorman PG and Rodriguez GC: Impact of progestin and estrogen potency in oral contraceptives on ovarian cancer risk. J Natl Cancer Inst 94: $32-38,2002$

6. Gao N, Nester RA and Sarkar MA: 4-Hydroxy estradiol but not 2-hydroxy estradiol induces expression of hypoxia-inducible factor 1alpha and vascular endothelial growth factor A through phosphatidylinositol 3-kinase/Akt/FRAP pathway in OVCAR-3 and A2780-CP70 human ovarian carcinoma cells. Toxicol Appl Pharmacol 196: 124-135, 2004.

7. Kimura A, Ohmichi M, Kawagoe J, Kyo S, Mabuchi S, Takahashi T, Ohshima C, Arimoto-Ishida E, Nishio Y, Inoue M, Kurachi H, Tasaka K and Murata Y: Induction of hTERT expression and phosphorylation by estrogen via Akt cascade in human ovarian cancer cell lines. Oncogene 23: 4505-4515, 2004.

8. Zhou HY, Pon YL and Wong AS: Synergistic effects of epidermal growth factor and hepatocyte growth factor on human ovarian cancer cell invasion and migration: role of extracellular signal-regulated kinase $1 / 2$ and p38 mitogen-activated protein kinase. Endocrinology 148: 5195-5208, 2007.

9. Bellacosa A, de Feo D, Godwin AK, Bell DW, Cheng JQ, Altomare DA, Wan M, Dubeau L, Scambia G, Masciullo V, Ferrandina G, Benedetti Panici P, Mancuso S, Neri G and Testa JR: Molecular alterations of the AKT2 oncogene in ovarian and breast carcinomas. Int J Cancer 64: 280-285, 1995.

10. Yuan ZQ, Sun M, Feldman RI, Wang G, Ma X, Jiang C, Coppola D, Nicosia SV and Cheng JQ: Frequent activation of AKT2 and induction of apoptosis by inhibition of phosphoinositide-3-OH kinase/Akt pathway in human ovarian cancer. Oncogene 19: 2324-2330, 2000.

11. Altomare DA, Wang HQ, Skele KL, De Rienzo A, Klein-Szanto AJ, Godwin AK and Testa JR: AKT and mTOR phosphorylation is frequently detected in ovarian cancer and can be targeted to disrupt ovarian tumor cell growth. Oncogene 23: 5853-5857, 2004.

12. MacDonald NJ, De la Rosa A and Steeg PS: The potential roles of $\mathrm{nm} 23$ in cancer metastasis and cellular differentiation. Eur J Cancer 31A: 1096-1100, 1995.

13. Tomic S, Ilic Forko J, Babic D, Sundov D, Kuret S and Andelinovic S: c-erbB-2, p53, and nm23 proteins as prognostic factors in patients with epithelial ovarian carcinoma. Croat Med J 44: 429-434, 2003.

14. Liu L and Yang K: A study on C-erbB2, nm23 and p53 expressions in epithelial ovarian cancer and their clinical significance. Zhonghua Fu Chan Ke Za Zhi 34: 101-104, 1999.

15. Galani E, Sgouros J, Petropoulou C, Janinis J, Aravantinos G, Dionysiou-Asteriou D, Skarlos D and Gonos E: Correlation of MDR-1, nm23-H1 and H Sema E gene expression with histopathological findings and clinical outcome in ovarian and breast cancer patients. Anticancer Res 22: 2275-2280, 2002.

16. Wang YF, Chow KC, Chang SY, Chiu JH, Tai SK, Li WY and Wang LS: Prognostic significance of $\mathrm{nm} 23-\mathrm{H} 1$ expression in oral squamous cell carcinoma. Br J Cancer 90: 2186-2193, 2004.

17. Wang LS, Chow KC, Lien YC, Kuo KT and Li WY: Prognostic significance of $\mathrm{nm} 23-\mathrm{H} 1$ expression in esophageal squamous cell carcinoma. Eur J Cardiothorac Surg 26: 419-424, 2004.

18. Hua KQ, Yao LQ, Cao Q, Huang Y, Zhao YQ and Feng YJ: Influence of estrogen and progestin on $\mathrm{nm} 23-\mathrm{H} 1$ expression in epithelial ovarian cancer cell lines via activation of phosphorylation signaling. Zhonghua Fu Chan Ke Za Zhi 41: 756761, 2006.

19. Brustmann H and Naude S: Expression of nm23-H1 in normal, hyperplastic and neoplastic endometrial tissues. Pathol Res Pract 195: 829-834, 1999.

20. Chen XF, Zhang HT, Qi QY, Sun MM and Tao LY: Expression of E-cadherin and $\mathrm{nm} 23$ is associated with the clinicopathological factors of human non-small cell lung cancer in China. Lung Cancer 48: 69-76, 2005.

21. Ohta S, Lai EW, Pang AL, Brouwers FM, Chan WY, Eisenhofer G, de Krijger R, Ksinantova L, Breza J, Blazicek P, Kvetnansky R, Wesley RA and Pacak K: Downregulation of metastasis suppressor genes in malignant pheochromocytoma. Int J Cancer 114: 139-143, 2005.

22. Whitley BR, Beaulieu LM, Carter JC and Church FC: Phosphatidylinositol 3-kinase/Akt regulates the balance between plasminogen activator inhibitor-1 and urokinase to promote migration of SKOV-3 ovarian cancer cells. Gynecol Oncol 104: 470-479, 2007. 